Revista Iberoamericana, Vol. LXXV, Núm. 229, Octubre-Diciembre 2009, 963-981

\title{
ESQUINAS Y/O ENCRUCIJADAS: \\ UNA MIRADA AL CARIBE URBANO EN MÚSICA Y LITERATURA
}

\author{
POR \\ Juan Otero Garabís \\ Universidad de Puerto Rico
}

Luego de una introducción con sonidos de sirenas, choques de vasos, una música de fondo y una voz que llama a un tal Avelino, la voz cantante de "Pedro Navaja" comienza su narración afirmando: "Por la esquina del viejo barrio lo vi pasar ..." (Colón \& Blades). Esta frase establece la perspectiva desde la cual la voz poética va a contar la batalla final de quien ha pasado a ser héroe de la cultura popular caribeña y latinoamericana: quien cuenta y canta mira desde un lugar cercano a la esquina, o desde ella. El poeta y el héroe visualmente se encuentran en la esquina punto imaginario en el que se topa el delincuente callejero con su cronista popular, como si allí también se encontraran dos o más culturas: la delictiva y la artística, la particular o local y la proveniente de tradiciones de muchísimas partes y, en la canción, la cultura oral y la letrada o en términos tradicionales y conflictivos, la alta cultura y la popular. No son culturas contradictorias, pero tampoco recorren ni se dirigen al mismo camino.

"Pedro Navaja" debe en gran parte su inconmesurable éxito a que narra de manera épica, con versos alejandrinos, una batalla cotidiana de las violentas ciudades caribeño-nuyorquino-latinoamericanas por medio de un personaje que también proviene de tradiciones literarias mezcladas con el jazz como el Mac the Knife brechtiano y armstrongniano. Este encuentro o cruce de tradiciones culmina cuando al final del episodio se asegura que "como en una novela de Kafka el borracho dobló por el callejón”. Esta esquina -la que por medio de otras referencias la canción sitúa en un barrio hispano de Nueva York-puede ser cualquiera de cualquier ciudad latinoamericana, y Pedro Navaja puede ser cualquier asaltante callejero de los sesenta o setenta, como si la esquina fuera un espacio emblemático de las culturas caribeñas y tal vez de las hispanas. Pero la forma de relatar esta historia estableció un lazo entre la literatura y la salsa como música popular y como "crónica del Caribe urbano", de tal manera que abrió las puertas para que esta "forma de hacer 
música” o esta "máquina” entrara a los estudios culturales en las universidades alrededor del mundo. ${ }^{1}$

Curiosamente, donde parece que más cerca están el narrador de esta épica popular y su héroe es cuando éste pasa por la esquina. La calle será el escenario para el resto de esta épica, incluso el de su batalla final con la prostituta. La esquina supone el encuentro de estos seres cuyas diferencias parecen más complementarias que antagónicas: sus caminos no apuntan hacia rumbos contrarios, aunque tampoco se dirigen al mismo destino. La esquina simboliza el espacio donde se encuentran sus historias, donde se cruzan la encrucijada, un punto decisivo en el camino, y el destino del héroe, quien opta por uno, no por quedarse en el cruce; así también este cruce representa el punto de observación del narrador-poeta-cantante, cuya decisión es la de asumir la palabra. Es como si la esquina recordara que las tradiciones provienen de orígenes diferentes y que bien pudieran juntarse hacia un mismo destino, cruzarse y compartir, o continuar rumbos diferentes, incluso actuar como si no se vieran. En la esquina, el artista parece protegido dentro del bar o cafetín, como un voyeur en la multitud, desde donde observa y canta al otro que pasa y se traslada para encontrarse como Edipo con la fatalidad del destino: "el que a hierro mata a hierro muere”. ¿Qué marca esta esquina: la distancia o la cercanía? $\dot{¿}$ Las diferencias o las semejanzas? ¿Es este cronista un observador externo: una especie de antropólogo urbano que se ha instalado en la esquina para realizar sus estudios? ¿ $\mathrm{O}$ es un nativo que ha aprendido los ritos de observación y descripción del científico social? ¿Es que la esquina pudiera simbolizar ese espacio caribeño en donde se cruzan tradiciones, donde el cronista popular se cruza con el antropólogo como personajes que vienen de caminos diferentes?

Este paseo crítico cultural tiene otro "punto de partida”: otra canción de Blades que vacila más con el concepto de la esquina como espacio emblemático de la ciudad sin atribuirle carácter épico ni trágico. Digo punto de partida entre comillas, porque la esquina no supone origen ni destino: más que una morada es un lugar de estar que pudiera referir al nomadismo sugerido por Deleuze y Guattari o la errancia de Glissant, pero cuya reiterada cotidianidad y similitud sugiere una permanencia mayor que la del nómada y, cuya recreación imaginaria en los discursos, en ocasiones la emparenta con la idealización del paisaje rural.

"Las esquinas son iguales en todos la'os" reitera el coro de esta composición de Blades, popularizada en Puerto Rico e internacionalmente (si es que este término aplica al mercado alcanzado por la Fania Records para mediados de la década de 1970) por Ismael Miranda (“Las esquinas son”). “En Panamá o en Borinquen sirven para estar paraos”, reza el primer estribillo de manera que detalla y caracteriza la

\footnotetext{
1 Me refiero a tres de las formas de referir y caracterizar a la salsa: Rondón; Quintero Rivera; Quintero Herencia.
} 
generalización normalizadora que establece el coro. La canción parece proponerse establecer a las esquinas como un lugar que emparenta las comunidades citadinas o pueblerinas del Caribe, partiendo de la comunidad Panamá y Puerto Rico, expresado por la letra y que la pareja del compositor y el cantante reiteran. El “todos la’os” que pudiera extenderse o entenderse como una experiencia de las urbes modernas, en especial las caribeñas y latinoamericanas como en "Pedro Navaja”, aquí parece circunscribirse al Caribe hispano, y el ritmo de "charanga-son” le sirve de marcador de fronteras. Pero más allá de delimitar la extensión de esta similitud, la frase destaca que la esquina sirve de lugar de encuentros cotidianos sin pretensiones de trascendencia: simplemente sirve para “estar para’os”. El masculino, que en español se usa como neutro, aquí es significativo, pues más adelante se referirá a los muchachos y a la “diversión [de] ver los pollos y su caminao”. Las esquinas son, pues, un lugar de encuentro y diversión para una comunidad masculina. Esta comunidad masculina coincide con un reiterado ambiente citadino: poste de luz, zafacón y "bonche”: "Encuentras el poste de luz y el eterno zafacón y el mismo bonche de siempre afincando un vacilón”. Una referencia a un perro que hace sus necesidades en "el mismo poste” y otra al paso de la policía proponen este espacio como una delimitación de territorios entre conducta popular y conducta ordenada, una frontera entre espacios “populares” y caminos oficiales. El estar en la esquina “afincando un vacilón” se asemeja al animal que delimita su espacio mediante el olor de su orina: un macho que desplaza a otro. El paso de la policía y el correspondiente azoro de los "habitantes” de la esquina ("La jara pasó a las doce y todo el mundo ya está asfixiao”) recuerda que la conducta ociosa y marginal es vigilada: que la esquina supone el cruce entre autoridades oficiales y autoridades marginales. La esquina, siguiendo a Michel de Certeau, sirve de escenario para desarrollar una conducta cotidiana que no sigue las normas de la ciudad ordenada desde las autoridades, por lo tanto para desarrollar las historias que De Certeau atribuye a los deambulantes, al mirarse su desplazamiento como actos delictivos (115-30). ${ }^{2}$

La esquina pudiera entonces considerarse como un espacio emblemático de la dinámica entre libertad y control que observa John Fiske en su análisis de la cultura popular:

The city is a mix of freedom and constraint. It is designed to promote certain ways of behaving, of moving, of thinking. It is the ultimate text, produced and reproduced by the forces of capital and law and order, designed for the most

\footnotetext{
2 De Certeau propone que mediante el acto de caminar el deambulante simula una fundación mítica del espacio como un ritual que "'provides space' for the actions that will be undertaken; it 'creates a field' which serves as their 'base' and their theater'” (124). Las palabras entre comillas son citas de Georges Dumézil. Idées romaines. París: Gallimard, 61-78.
} 
efficient exercise of what these forces believe should constitute everyday life. Yet its very complexities make it also the place of great disorder, its multiple systems of control and discipline open up gaps where life can be lived out of control, beyond discipline. (204)

La calle es para la ciudad ese lugar de apertura donde coinciden y confligen esos sistemas múltiples de control y disciplina con los "huecos" o "abismos" de libertad para conductas que exceden ese control. En este sentido, si la calle está diseñada como vía de tránsito, la esquina representa la intersección de al menos dos vías que atraviesan la ciudad y, por lo tanto, cruce y/o encuentro de sujetos que transitan en diferentes direcciones a ritmos similares o diferentes. Sin embargo, las esquinas de Blades son un espacio de estar, actuar y ver, y no de transitar: una conducta contraria al tránsito para el cual está diseñada la esquina como cruce de caminos. ¿Qué sugiere entonces este estar en la esquina en vez del pasar por la esquina? ¿Es una negativa al cruce, al pasar al otro lado, a continuar el camino, a proseguir su destino? ¿Es convertir el lugar de tránsito en espacio de ocio? El ocio o la diversión, según Fiske, constituye una actividad de evasión a la conducta normativa de la sociedad productivista, que desea y necesita ciudadanos insertos en la producción y el consumo de mercancías. Esta visión utilitarista ve la necesaria masa de desempleados como fuerza de presión a los trabajadores. Insistir en privilegiar el ocio, el "estar parao" "afincando un vacilón”, equivale a privilegiar en el orden existencial la diversión y la "inacción” frente a la ética productivista y laboral. Equivale entonces a alterar la historia del trabajo y del desarrollo planificados desde la ideología dominante en una evasión en la que cada transeúnte "escribe" su historia y hace de un lugar su espacio. Según Michel de Certeau:

A space exists when one takes into consideration vectors of direction, velocities, and time variables. Thus space is composed of intersections of mobile elements. It is in a sense actuated by the ensemble of movements deployed within it. Space occurs as the effect produced by the operations that orient it, situate it, temporalize it, and make it function in a polyvalent unity of conflictual programs or contractual proximities. [...] In short, space is a practiced place. Thus the street geometrically defined by urban planning is transformed into a space by walkers. (117)

La contradicción que supone la canción de Blades es la de convertir el lugar de tránsito en el espacio de estar; la historia del pasar o trascender en la del quedarse. En ese espacio, muy disimuladamente, pero en un gesto que pudiera reconocerse como el de asociarse con la jaquetonería del macho caribeño, una voz diferente a la de Miranda se cuela entre el solo de tres para decir: "Cuidao con el paquetico que está botao en el piso”. La insuficiencia lingüística se une con el eufemismo 
para ocultar a qué refiere el susodicho paquete que por la situación y la precaución aludida pudiera estar refiriéndose a una transacción ilegal de drogas. Esta alusión, que asocia el estar en la esquina con la actividad ilegal, subraya la libertad que este espacio presenta frente a la legalidad del orden establecido para el que la presencia de la policía representa un corte del espacio vital, dándole un sentido más literal a la frase: "La jara pasó a las doce y todo el mundo ya está asfixiao".

Dos soneos sueltos al final de la canción recuerdan que la esquina también puede ser lugar de poder o de autoridad. "El bochinche" y "el comentario" convierten a la esquina en un espacio cuya verbalidad origina rumores, historias y mitos que pueden ser represivos y autoritarios. En el afamado cuento de Luis Rafael Sánchez, “iJum!”, el rumor de la comunidad se presenta como voz hostigadora: "El murmureo verdereaba por los galillos. [...] en cada recodo, en cada alero ..." (49). Significativamente, los hombres participan de esta hostilidad desde la esquina: “En cada esquina, los hombres se vestían la lengua con navajas” (51). Una canción del dominicano Wilfrido Vargas hace referencia al temor que causa el qué dirán de la esquina, aunque sin aludir a que se trata de voces masculinas necesariamente, cuando quien recién inmigra teme "que no me digan en la esquina 'El venao, el venao' que eso a mí me mortifica”. Las voces masculinas ejercen su autoridad y su opresión desde la esquina, como si este espacio fuese su fortaleza, su palacio, su corte o su morada. Ancladas en su esquina, las autoridades de la marginalidad callejera reprimen y desplazan al homosexual y al cornudo mediante "el comentario" y "el bochinche” que no parecen amenazadores en la canción de Blades y Miranda.

"Las esquinas son" no propone la esquina como espacio privilegiado en la dinámica orden/desorden citadinos. Por el contrario, parece un espacio intrascendente, en vez de trascendente, pero en el cual subsisten, sobreviven, merodean o habitan "los muchachos". Curiosamente al final, al acelerarse el ritmo de la canción, el sonero (cantante), Miranda, altera la contemporaneidad entre espacio y voz poética, establecidos por el presente verbal, y contempla las esquinas desde otro tiempo: “iEh los muchachos! / Aquellos tiempos / en que bailábamos / en las esquinas”. ¿A qué obedece esta aparente mirada nostálgica de la voz poética? ¿Por qué alterar el presente de las esquinas (“son”), por un tiempo en que "bailábamos”? El son de las esquinas se traspasa del verbo copulativo al ritmo: las esquinas se convierten en el son como intensificación rítmica de la charanga con que comienza y como canción que evoca un pasado. Desde la gozosa celebración rítmica se construye una visión nostálgica de la canción. El acelerado ritmo de la ciudad convirtió el presente de la esquina en un aparente recuerdo de juventud: era en otro tiempo en el que se "afincaba" el vacilón. ¿Y ahora no? ¿Es que la esquina ya ha dejado de ser espacio de estas historias de estar? ¿O es que simplemente la "voz poética" se hace eco de la "tradición" literaria puertorriqueña para la cual la nostalgia es la 
estrategia para construir íconos culturales como el campo y el campesino? Esta vez una nostalgia por la propia juventud, pues la esquina se presenta como el lugar de "los muchachos", de sus años de juventud.

Para la misma época en que Miranda grabara la canción de Blades, el Gran Combo grabó con Andy Montañez como vocalista dos canciones dedicadas a ciudades: “Un verano en Nueva York” y "El son de Santurce”. Las canciones de esta agrupación, al contrario de muchas composiciones de Blades y de Catalino Curet Alonso, no se destacan por "ser crónicas urbanas": "Un verano en Nueva York", composición de Justi Barreto, es buen ejemplo. A diferencia de "Pedro Navaja" y de los temas de Willie Colón y Héctor Lavoe, Nueva York en esta ocasión es un lugar de veraneo. La mirada que se extiende es de un boricua que visita a sus amigos del otro lado del charco, quienes lo llevan de excursión por algunos de los lugares de la ciudad. No hay descripción de la vida cotidiana en la ciudad y la experiencia se limita al goce turístico con un poco de color étnico, pues entre las atracciones figuran el "Desfile puertorriqueño," una "fiesta folclórica en el Parque Central” -alusión a las reuniones que la comunidad puertorriqueña acostumbraba a realizar los domingos en la parte noreste del parque durante finales de la década de 1960 y comienzos de la siguiente- y "la fiesta de San Juan”.

"El son de Santurce” resulta más interesante y pertinente para propósitos de esta mirada. La composición de E. Vigoreaux presenta una visión panorámica y paisajística de lo que se caracteriza a partir de mediados del siglo xx como uno de los centros urbanos más característicos de Puerto Rico: "Santurce es la bella tierra, / rodeada por los mares / dorada del sol de oriente / y dormida por sus palmares". Esta versión paisajística del "centro" urbano contrasta o se complementa con las palabras del cantante y sus soneos. En primer lugar, desde el comienzo de la canción la voz del intérprete inicia un viaje: "Y voy pa’llá"; posteriormente su descripción refiere a lugares de tránsito: "el puente Dos hermanos” y "la veinticinco y su callejón”; y, por último, la referencia a su cuna destaca por estar definida por el trabajo: Trastalleres. Lo que para el compositor pareciera ser un paisaje delimitado por las costas y las palmeras, la voz cantante lo ubica entre puentes y paradas del tren. La ocasión festiva que motiva la letra -"Hoy tus hijos están de fiesta"- la voz cantante la sitúa en la cotidianidad del trabajo al destacar que "en Cantera se oye Paleco con su pregón: 'Mantecadito, mantecadito, mantecadito, manteca mantecadón'”. "El son de Santurce” se convierte así en un espacio donde se encuentran la mirada paisajista del compositor y la urbana del cantante como dos experiencias, dos perspectivas y dos celebraciones marcadamente diferentes del "orgullo" citadino, miradas que se funden significativamente en el último soneo cuando se alude a "El son de la loma”, la famosa canción del Trío Matamoros que describe al género musical en un viaje de origen indeterminado entre la loma y el 
llano. Esta morada, esta reconocida cuna de origen -“yo nací en Trastalleres y por eso tú eres mi amor”-, es descrita y resaltada por sus vías de tránsito, así como Nueva York es también espacio para recorrer "festivamente" como señal de la alegría del encuentro de las dos comunidades puertorriqueñas: la de aquí y la de allá. ¿Es Santurce como una gran esquina? ¿La más famosa como aludía una de sus tiendas: La Esquina Famosa?

El contraste entre la contemplación paisajística natural y la cotidianidad y el tránsito citadino, no necesariamente niega la primera, sólo parece otro momento u otra circunstancia. Mediante la mirada contemplativa de los mares, el sol y los palmares se insinúa una contemplación relajada desde el lugar hacia afuera, que curiosamente parece aislar el lugar de la comunicación con los territorios aledaños o el tránsito hacia ellos. Sin embargo, las intervenciones de Montañez de inmediato trazan las colindancias del puente Dos Hermanos, donde comienza el islote de San Juan y la (parada) veinticinco, casi al final de la colindancia de Santurce y Hato Rey. Se elude mencionar la laguna del Condado y el Caño Martín Peña que serían los límites naturales de Santurce y se elige, en cambio, referir vías de comunicación: puente y parada de tren. Al mismo tiempo, se presenta la pertenencia al lugar por el nacimiento: Santurce es un lugar donde los trabajadores asientan "sus raíces". Santurce es simultáneamente origen, morada (raíz) y tránsito. La comunidad se comunica con sus territorios aledaños sin dejar de afirmar el orgullo por su origen, su particularidad. Santurce adquiere cierto carácter de espacio fronterizo y de espacio transitable desde el cual se comunica hacia otros espacios de la ciudad y por el cual se pueden intercomunicar; se define entonces más que por su alegada particularidad -el aislamiento en el que la sitúa la letra de Vigoureaux- por su relación entre otros espacios y otras comunidades. ¿Es que los santurcinos se ven como "vecinos” y como "viajeros”? ¿Es Santurce una morada, un camino y un cruce? ¿Hasta dónde extienden sus raíces los habitantes de Santurce? Al mirar "El son de Santurce” junto a "Un verano en Nueva York” se aprecia la cultura puertorriqueña en tránsito por calles y parajes del área metropolitana y de viaje: desde los palmares y Trastalleres hasta el Parque Central la cultura puertorriqueña desfila, celebra y transita como si construyera sus historias en un quehacer constante en el que destacan la cotidianidad, la familiaridad, la música y la fiesta. En esta morada y por su errancia, la cultura puertorriqueña deja a un lado la imagen del árbol y de las "raíces ancladas en la plata de los Andes”, con las que Martí proyectaba las culturas hispanoamericanas, para pensarse en términos más rizomáticos y nómadas, cuyas comunidades cargan su cultura y sus raíces y las reimplantan y reconstruyen en otros espacios. 
El Caribe COMO encrucijada

Una mirada a los estudios sobre el Caribe no puede evitar reconocer la constante referencia a esta relación de cruce, de morada y de movimiento. Por ejemplo, el ya legendario y canónico libro de Antonio Benítez Rojo La isla que se repite describe el desplazamiento de las múltiples migraciones y el contrapunto entre plantación y contraplantación como una constante en los diversos países de un Caribe que se extiende, según el crítico cultural, desde la ciudad de Bahía, en Brasil, hasta Nueva Orleáns, en Estados Unidos. Dimensión que se extiende aún más, según el barbadino George Lamming para quien: "There is a Caribbean World that exists, in a very decisive kind of way, in many metropolitan centres, whether in North America or Europe" (citado en Torres-Saillant 112). El movimiento tanto circular como contrapuntístico y el desplazamiento son aspectos que le dan su particularidad a una región o zona cultural formada por migraciones continuas. En este sentido, la descripción que hace Édouard Glissant resulta bastante acertada. Para el crítico y escritor martiniqués, el Caribe "has always been a place of encounter and connivance and, at the same time, a passageway toward the American continent” (33). Por eso, para Glissant, el Caribe ilustra, así como es ilustrado por lo que él llama la "poética de la relación”, pues su definición, su característica y su formación se entienden mejor desde la perspectiva que considera las mezclas y diferencias, tanto como las proclamadas purezas o raíces ancestrales y similitudes.

Una de estas relaciones complicadas es la de pensar el Caribe como espacio de enraizamientos y/o de errancia, pues el Caribe es una morada y un pasaje; para muchos una morada transitoria, que pudiera significarse como la estadía en un hotel. Esta semejanza la desprendo del sugestivo ensayo de James Clifford, “Traveling Cultures”, quien al replantear la relación entre el viaje del antropólogo y la morada del nativo como sujeto estudiado sugiere la metáfora del hotel como espacio que "frame[s], at least, encounters between people to some degree away from home" (105). Compuestas por migrantes voluntarios o forzados de otros continentes, las culturas caribeñas se forman mediante las mezclas de culturas provenientes de otras partes en su transculturación en estas tierras, como las pensaba Fernando Ortiz. De manera que el Caribe es la esquina, más que el camino o la morada, donde se encuentran dichos viajeros; esquina que para algunos se transforma en puerto y morada y para otros simplemente en puerto y pasaje.

La cita de Glissant sugiere también mirar al Caribe como encrucijada. Sin embargo, esta perspectiva enfatiza la necesidad de trascender las coyunturas y decisiones que un cruce representa. A pesar de que la decisión de quedarse en el Caribe es vital para la formación de sus culturas, mirarlo como errancia y rizomáticamente favorece la decisión de pasar, de elegir uno de los caminos que 
la encrucijada ofrece. Considerado así, el Caribe es una encrucijada en la cual el sujeto no puede permanecer, al menos por mucho tiempo; ante ella debe optar por un camino y proseguir su destino. Por ejemplo, una de las novelas que de cierta manera fundan la narrativa del Caribe anglófono, In the Castle of My Skin de George Lamming, presenta la esquina, o mejor, el pasarse y/o quedarse en la esquina como peligro de fracaso para el joven $\mathrm{G}$. En un pasaje significativo su madre se lamenta de la insistencia de Bob, su vecino, de janguear en la esquina:

An' all I talk to the boy about that corner he won't hear. Mornin', noon and night I pray and I preach to the boy. I tell him what the worl' is like an' what he must expect, an' the boy won't hear, won't hear, won't hear not a single word I say. I don't say you mustn't play, I don't you mustn't have friends, but that corner is no good for you. I tell you repeatedly, once, twice, three times, I tell you to choose. Either you go with the gang at the corner, obey them, do as they tell you to do and live as they live and don't let me waste my time, or you do what I say. You can't serve two masters. I know what the old people say is true. You carry a horse to the pond but you can't make it drink, an' if you've no mind, then there's no use my tryin'! All my labour will simply go into Maxwell pond. The mind is the man, That's all I have to say, an' I'm tellin' you, I'm tellin' you once, twice, for the third and last time I tell you, if I catch you at that corner again, I'll roast your tail alive. (113)

La esquina no sólo es un espacio que amenaza el futuro del protagonista en su novela de aprendizaje -el "otro 'maestro'”- sino que se torna en una especie de rito de iniciación por el cual éste debe pasar para adquirir el conocimiento que lo convierta en un hombre maduro. La esquina es el espacio en el que la "ganga" de jóvenes desafía no sólo el orden de la comunidad, sino el futuro que sus familias planifican y desean para ellos. El reto para los jóvenes, según la perspectiva de los adultos, es no quedarse en la esquina, superar la adolescencia. En la novela de Lamming la esquina es otra encrucijada en la que el sujeto se puede perder si no la cruza o la atraviesa.

En una de las obras cumbres del teatro del santalucino Derek Walcott, Dream on Monkey Mountain, también la esquina como encrucijada tiene un aspecto significativo. Como recuerda Lowel Fiet: "The 'journey' is one of the principal tropes of twentieth-century drama [...]. Similarly the 'crossroads' corresponds to the dramatic itself: conflict, crisis, climax, and resolution -complete, partial, or frustrated- before the journey can end or resume" (104). La crucial escena III de la primera parte toma lugar precisamente en un mercado en un cruce al amanecer. La hora del día y el lugar corresponden a momentos y espacios de cambio, y el mercado es el espacio del intercambio. Sin embargo, los intercambios que ocurren 
en la escena son más simbólicos que materiales. Como profeta del renacer del sueño africano, Moustique anuncia la llegada de Makak: "Makak have come to Quatre Chemin Market and neither corporal nor spiritual stopping him today" (266). Anuncia también que ésta será la última vez que el místico Makak atravesará ese camino: "because Makak shall not pass this road again. His dream call him to the sea, to the shore of Africa” (267). Moustique y Makak en la obra son la reencarnación del mito del origen y de la trascendencia o retorno al lugar de las raíces, África. Como señala Nana Wilson-Tagoe, de esta forma Walcott vincula su obra con el tiempo inmaterial y universal del mito (173). Sin embargo, la encrucijada del pase o regreso definitivo al lugar de las raíces marca el fin de Moustique, quien es asesinado por la multitud, así como el trascender de Makak por vía del sueño. Quienes no acceden a la trascendencia mítica se quedan: los oficiales gubernamentales y los vendedores del mercado en la esquina.

Diferente es la perspectiva que en 1988 se presenta en el álbum Antecedente de Rubén Blades. En esta grabación el cantante y compositor panameño traza un retorno nostálgico y sentimental hacia Panamá, país al que se le dedica la grabación, mediante la memoria de sus barrios y sus puertos. Uno de los temas más "patrióticos" del disco, "Nacer de ti", composición de Franco Nieto, define ese nacer como un viaje: "Vengo del mar y la angostura" son las primeras líneas de la canción, "cruce del camino y de la tarde". Esta voz que afirma su fidelidad y su orgullo patrio se describe por la traslación y no por la raíz -viene del mar-y se define por su mirada a los barcos y su relación con los viajeros: esperando amistosamente al que llega y despidiéndose con dolor del que se va. En otra canción, "Noches del ayer", la voz poética es la que viaja y se define por el reencuentro con estos barrios y su regreso: "Aunque estoy por otras tierras, ya pronto vuelvo. / allá, por el 5 de mayo los voy a ver".

El definirse por el viaje también requiere una referencia al pasar del tiempo: la voz poética afirma provenir de la tarde y también "del tiempo tuyo y del tiempo mío”. La referencia al cruce y al crepúsculo acentúa esa mirada de esos intermedios, esa encrucijada, desde la que se desea que los viajeros se queden: vengo "de los que llegaron, del que no se fue”. La reafirmación del viaje y la procedencia -la palabra "vengo" inicia tres de los once versos de la canción-se une con un haberse quedado, con ese que no se fue, o una partida, tal vez la suya, que le arruinó los sueños: "Mis amores se agrietaron y mis sueños sucumbieron en la estela de los barcos”. ¿Se añora al amor y los sueños desde la nave o desde el puerto? El cruce supone tanto encuentros como partidas. ¿También dos tiempos? Este cruce de tiempos se puede apreciar de maneras más significativas como cruces entre la modernidad y la premodernidad en otro de los temas del disco,“Contrabando”, el que se analizará más adelante. 
La tónica de la canción sienta la apariencia del puerto y la tierra que recibe al viajero, pero el que se queda también ha venido de otra parte y de otro tiempo. De este modo se define el nacer como una proyección del deseo, como un fundirse con una raíz que no se siente segura. Quien llegó y se quedó quiere asentarse, quiere "nacer de ti". Sin embargo, a pesar del título de la canción, esta no hace referencia a la cuna, la familia ni el nacimiento. Aunque sí es evidente el deseo de "ser la tierra". La canción tiene dos coros, el primero reafirma el viaje y la procedencia al repetir las dos primeras líneas de la canción; durante este breve coro las intervenciones del solista refieren a la hospitalidad y el cariño con que se espera y se recibe al viajero: "Soy el abrazo que espera / al que quiera regresar / soy la mano saludando / al que acaba de llegar”. La segunda intervención del solista funde la voz de este "viajero" con la tierra: "Soy viento, sol y palmera / luna, selva, cielo y mar / río, llano y cordillera / y me llamo Panamá”. El "clip” turístico no puede ser más claro: el sujeto que espera al viajero/a está fundido con el paisaje. Durante los soneos bajo el siguiente coro se afirmará que desea "ser la estrellita de mi bandera". Esta simbiosis es subrayada con el segundo coro: "Nacer de ti, ser la tierra / y el que se queda. Nacer de ti”. El infinitivo sugiere que el "nacer" no es un hecho sino parte del deseo: se desea nacer de ti, así como se desea ser la tierra y el que se queda. Este puerto, este cruce entre dos mares, se define por el deseo de retener viajeros, contrastado con la ansiedad del viaje. La respuesta del solista reitera el viaje: "ser el camino del que regresa”. El que regresa es quien inició o continuó el viaje, quien se trazó futuros, sueños, en el viaje mismo o en otras partes. El regreso supone una de tres alternativas: que se reconoce la futilidad de esos sueños; que se fracasó y que esta tierra lo espera y lo recibe; o que el viaje nunca se pensó como para toda la vida, sino por motivos de los “cabotajes”, la "fiebre y el oro que pasó” y/o de la rebelión, "el cimarronaje”. Como ciudad puerto, Panamá se define por ese intercambio entre viajeros y residentes: entre los que llegan y los que se queda(ro)n.

La expresión “nacer de ti” devela el deseo de nacer de nuevo, íntegramente de esa tierra: fundirse con su naturaleza y su gente. Pero ¿cómo ser de un lugar y venir de otro? ¿Qué ha pasado con el bagaje del “otro lugar”? ¿Se propone romper una memoria y que otra sea la que perdure? Panamá se define por los cruces, encuentros y despedidas de los puertos: la tierra que saluda al que llega y al que regresa y el amor o el deseo porque se quede. ¿Porque se quede a esperar a otros y a otras? Esta percepción de Panamá intenta definir la tierra como el cruce y la encrucijada: el pasaje y la estadía. Singularmente, la define desde la mirada del que se queda, de quien añora que otros vengan, regresen y se queden como él. Este puerto es un cruce, un espacio donde unos se quedan y hacen sus historias, muchas veces observando a quienes van y vienen: esperándolos y extrañándolos. El sueño por una tierra que está al otro lado del Atlántico de la obra de Walcott aquí se traslada 
al puerto caribeño: el deseo es hacer de este cruce el lugar de origen, de nacimiento; aunque este sea una esquina donde unos "están paraos” y otros pasan.

Quien se traslada por vía del sueño es Blades, que ha hecho su carrera y ha ganado fama fuera de su país. Blades es quien con esta grabación "regresa” a los motivos panameños con referencias a sus personajes y hechos históricos, sus plazas, sus calles e incluso sus seres cercanos. Regreso que puede leerse como el de un contrabando, pues la canción que le sigue a "Nacer de ti”, "Contrabando", se refiere a un indio que vende artículos importados viajando en canoa por el Orinoco entre la selva. Los artículos resultan llamativos, pues contrastan con la naturaleza:

Carga cerveza importada. Trae veinte pantys franceses.

Trae Malboro americano y tres radios japoneses. Mentolatum y chingongo. Collares de fantasía.

Postales del Papa de Roma. Seis “Playboys” y baterías.

Sus clientes van desde el alcalde y el sacristán hasta los guerrilleros. La escena parece tomada de una novela de García Márquez, con quien Blades había cruzado sus melodías en una producción anterior, por el contraste entre los artículos importados como signos del comercio y del avance, en canoa, del consumo de la modernidad, con la naturaleza y las poblaciones de vida casi premoderna. La canción supone un viaje y un cruce de tiempos: significativamente el contrabandista es indio. Quien trae los productos extranjeros de la sociedad industrial es el que según los supuestos de la ideología indigenista encarna los valores de la naturaleza y de la patria. ¿Por qué este cruce? ¿Por qué alterar los elementos? ¿Por qué el indio vive de vender "Malboro americano", "radios japoneses”, "Playboys” y "pantys franceses” y no del cultivo y el trabajo de la tierra? ¿Ha sido su cultura suplantada por los productos manufacturados en el extranjero? $¿$ El desplazamiento de su tierra nativa lo ha convertido en nómada y contrabandista? En cambio, quienes habitan la tierra parecen pertenecer a la modernidad occidental: son representantes del orden de los sistemas políticos y religiosos impuestos por la conquista europea y sus consecuentes oposiciones macropolíticas: el alcalde, el sacristán y los guerrilleros. El nativo que fuera sujeto de estudios antropológicos es presentado como un comerciante semimoderno: un comunicador entre asentamientos de la modernidad. Este indio sería un sujeto que trastoca los estudios antropológicos, tradicionalmente interesados en poblaciones asentadas en territorios no modernizados y que se enfocan en "sus raíces" culturales. El contexto americano que media el cruce entre las culturas que llegaron de Oriente, en este caso es el río más que la selva. Aquí no hay lugar de sueños por una tierra o una pertenencia ancestral ubicadas al otro lado del mar: la cultura, expresada por este caribeño continental o intercontinental, Blades, es una 
que transita y comercia. Y en este caso, el contrabando del nativo también refiere a la venta del disco como otro producto extranjero: el cual construye sus antecedentes y se vende: ¿como producto comercial importado o como representación de las raíces culturales?

La contemplación de los viajes y las rutas se aprecia en dos de los trabajos más recientes sobre el Caribe: Caribe Two Ways de Yolanda Martínez-San Miguel y An Intellectual History of the Caribbean de Silvio Torres-Saillant. En suvalioso y amplio estudio de las rutas de las diásporas del Caribe hispano en su multidireccionalidad, Martínez-San Miguel observa cómo las culturas nacionales son atravesadas por los viajeros en dos direcciones: desde y hacia los puertos de origen. Igualmente, en la ruta emancipatoria del pensamiento caribeño que traza Torres-Saillant abundan las referencias a contrapuntos y viajes. Según este pensador, el Caribe se forma "en relación" con occidente como centro cultural ante el cual tiene que declarar su humanidad, su autonomía de pensamiento e independencia cultural. En este libro la inteligentsia y el pensamiento caribeño -como ontología y como forma de verse a sí mismos- viajan desde el descubrimiento, la conquista y la dominación hasta el cimarronaje, pasando por los contrapuntos de alteridad, de dominación e identidad. Mirando a gran parte de la intelectualidad caribeña como homo migrans, la experiencia del viaje y la migración entre dos puertos, dos espacios o dos tiempos imaginarios, resulta determinante para este teórico dominicano.

Resulta interesante el contrapunto que se puede desprender de ambos libros. En primer lugar, el privilegio del viaje le resta peso a la insistencia en las raíces y las tradiciones, que cada vez se ubica más en estos tránsitos. Sin embargo, en segundo lugar, ¿qué pasa con los que se quedan? ¿Por qué el quedarse, el no atravesar el cruce, el "estar para'o" no son contemplados como alternativas? Y tercero: ¿cuántas vías, cruces y estadías supone el viaje de un/a dominicano/a que migra en yola a Puerto Rico, donde se queda por un tiempo antes de continuar viaje a Estados Unidos? ¿ $\mathrm{O}$ un cubano y/o haitiano que a esa ruta le añade la estadía en República Dominicana?

Para intentar aproximarme a estas diatribas quisiera echar una mirada al cruce entre lo cotidiano y lo trascendental que supone mirar las esquinas urbanas vis à vis los cruces de caminos: es decir, encontrar los estudios de la ciudad y las prácticas de la cotidianidad con las creencias ancestrales de las tradiciones culturales del Caribe. Mirar el Caribe como encrucijada conlleva pensar en Eleguá como deidad que la rige. Esta deidad, a quien primero se le debe rendir ofrenda, es la que rige los caminos y las encrucijadas. A su vez, según Henry Louis Gates, es la que infiere en la interpretación, la ambigüedad, los cruces entre la oralidad y la escritura y las diferencias. Eleguá, quien en las tradiciones afrocaribeñas y afroamericanas asume diferentes nombres, es el intermediario entre los seres humanos y los orishas, pues 
es quien interpreta la palabra sagrada, el Ifa, para quien no la conoce. Al tiempo que guía los caminos, Eleguá es también una deidad engañosa, un truquero. ${ }^{3}$

Gates privilegia su regir sobre la interpretación y su carácter ambiguo para presentarlo como deidad de los críticos y del modo de significar de las culturas afroamericanas. Recuerda, además, el crítico afroamericano que "Esu’s often stated dwelling at the crossroads is [...] at the crossroads of differences; there is no direct access, or contact, with truth or meaning ...” (41). Eleguá habita en el cruce como encrucijada de diferencias, en las cuales no hay acceso directo a la verdad. ¿Qué significa habitar o morar en el cruce? Se insinúa una inestabilidad entre el estar de la deidad y del cruce como pase y como selección entre caminos. La cultura afroamericana al tiempo que le concede importancia a la encrucijada como umbral del destino, para cuya decisión se requiere de la deidad, parece restarle peso cuando la deidad que guía y que descifra los signos y el lenguaje es ambigua y engañosa.

Quizás entonces, para mirar esas encrucijadas sea pertinente recordar las prácticas de la vida cotidiana, según De Certeau, y los huecos que la cultura popular representa frente a la disciplina de la ciudad como espacio ordenado, según Fiske. La libertad se ejerce tanto mediante el desplazamiento por las calles como mediante el no cruzar las esquinas. Como se puede apreciar en "Las esquinas son” y en In the Castle of My Skin, estar en la esquina es visto por las autoridades como un desafío al orden. Pero ¿qué hacen estos transeúntes frente a las encrucijadas de la ciudad? ¿Pasan como Pedro Navaja o se quedan "para'os” como "los muchachos”? ¿Prosiguen su destino trazando sus espacios y sus historias o cantan, poetizan o analizan las historias de los otros? ¿Es el quedarse, el no pasar, atravesar el cruce, ni doblar la esquina, otra forma de escribir una historia? ¿Ésta es la historia de los que se quedan en el Caribe? ¿Es el Caribe el espacio para las historias de Eleguá? ¿O los caribeños asumen el privilegio de Eleguá de "hacer lo que le venga en gana" y de quedarse en la esquina, el puerto, el cruce y la encrucijada? ¿No atravesar la esquina, la encrucijada, es no trascender? ¿Sería mucho pensar en el Caribe como pasaje cuya trascendencia es atribuida a quienes lo atraviesan, mientras que los que lo habitan están condenados a la intrascendencia?

Dos pensadores y literatos puertorriqueños distanciados -pero emparentados, según Juan Gelpí-Antonio S. Pedreira y Luis Rafael Sánchez, critican la confusión, la vacilación y la oscilación de los puertorriqueños al no asumir la ruta de su destino. En 1934, Pedreira criticaba que: “[e]n instantes de trascendencia histórica [...] cuando el gesto viene empapado de oleadas de sangre africana quedamos indecisos, como embobados ante las cuentas de colores o amedrentados ante la visión cinemática de brujas y fantasmas” (50). Mientras que en La guaracha del

\footnotetext{
3 Para una recopilación de mitos sobre Eleguá véase Arce Burguera y Ferrer Castro.
} 
macho Camacho hay una crítica implícita a la vez que un goce del vacilar como relajo y como indecisión, en su ensayo canonizado "La guagua aérea", Sánchez observa que "La guagua aérea oscila entre el tumulto y el peso de la quimera, entre el compromiso con el salir adelante y la cruz secular del Ay bendito" (15). Más adelante se describe a "una adolescente" que "no sabe a qué rayos va". A pesar de que, como señala Gelpí, en su novela, Sánchez "desplaza el clásico de Pedreira”, "presenta ... un relevo de tropos" y "se da paso a otro tipo de literatura", en su "relación dialógica” Sánchez sostiene las mismas metáforas del viaje: la vacilación y la indecisión como "taras" a superar por la población puertorriqueña, aunque sin atribuirlos a la herencia africana como Pedreira. A ello las canciones de Blades y Miranda parecen responderle: “¿Por qué? Si en esa intrascendencia de la esquina, ‘el mismo bonche de siempre’ es feliz ‘afincando un vacilón””.

Miradas y CHAMACOS EN LAS ESQUiNAS

Dos textos de la literatura puertorriqueña nos ofrecen una perspectiva desde la cual quizás se pueda cerrar este jangueo por las esquinas caribeñas. En su más reciente novela, Nuestra señora de la noche (2006), Mayra Santos Febres contrapone la esquina como espacio de alternativas y búsquedas marginales frente a la crisis económica y los conflictos sociales. En una escena que sugiere la decisión de la joven Isabel para comenzar el negocio que le abrirá los caminos del éxito, aunque en los márgenes de la economía y la sociedad sureña, camina por las calles de Ponce durante el periodo de la depresión económica y la prohibición. Se encuentra con su anterior patrono, Don Antón, quien se lamenta del impacto negativo que la crisis económica ha causado en su sastrería. Ambos contemplan la plaza, en donde unos manifestantes escuchan al negro Demetrio Sterling, amigo y tutor de infancia de Isabel. Don Antón culpa a los trabajadores cuyas huelgas afectan la economía y su negocio. La joven Isabel, quien ha aprendido con el viejo Demetrio acerca de luchas obreras y feministas por medio de las lecturas de Luisa Capetillo, ni secunda la queja de su ex patrón ni se detiene a escuchar a su antiguo tutor. Prosigue su camino y encuentra al sargento Peña a quien le paga por proteger su tráfico de alcohol clandestino mientras vigila desde la esquina a los manifestantes. Al pasar, porque "la vida seguía", Isabel se percata de las miradas en las esquinas:

Mientras tanto, en las esquinas, miradas ojerosas esperaban con ansia que alguien las llamara para desempeñar cualquier trabajo: barrer la acera, limpiar las baldosas de entrada, desempacar en las estanterías. La calle está llena de correcostas. La mayoría eran mujeres, muchachitas recién bajadas de los campos que buscaban emplearse en cualquier cosa. Cuando no tenían otro remedio, se vendían ellas mismas por algunos centavos. A Isabel le recordaban a ella, o lo que pudo haber 
sido ella sin el pitorro y sin los dineros extra que ganaba con el licenciado. Isabel sabía que aquéllas trabajarían hasta por comida, se vendería [sic] a los dueños de tienda por una esquina seca donde dormir. Que aunque tuvieran el estómago vacío, la irían a buscar por referencia para comprarle un traguito de ron. Ganas tenía a dárselos de gratis. “Tú estás loca, mujer, ¿y nosotras qué?”, le recordaba Leonor. “Ponte dura, Isabel”, pero no lo lograba. No del todo. (255-56)

El momento de crisis supone una encrucijada para Isabel. Ya no es ni la costurera de don Antón ni discípula de Demetrio. La calle es su espacio, allí "sigue viviendo”, desde y por allí realiza su negocio. La esquina es el lugar desde donde la autoridad vigila a los opositores al orden mientras acepta las propinas de los corruptores; también es el lugar desde el cual se busca una oportunidad. Son las miradas de estas “muchachitas” las que sirven de agente catalítico para la idea del otro negocio de Isabel: el Elizabeth’s Dancing Place. Se sugiere que los negocios de Isabel son un cambio de camino que evade la confrontación que la lucha macro política de los huelguistas supone. La mirada por la esquina le sugiere el negocio que se convertirá en un espacio de poder alterno al orden oficial: el poder de la marginalidad negra y femenina.

La reflexión más interesante que conozco sobre y desde la esquina en la literatura puertorriqueña la provee el “más famoso poema” de José Raúl González, “Gallego”: “Chamaco’s corner”. Este poema no sólo emblematiza la cultura masculina y juvenil contemporánea, sino que presenta un interesante contrapunto que complementa la perspectiva de “Las esquinas son”. A diferencia de la canción de Blades y Miranda, el poema de Gallego comienza presentando la esquina como un lugar conflictivo y de violencia: "Los chamacos no se van de la esquina ni a tiros” (65). De ahí en adelante el poema comparte elementos de vida cotidiana, conducta delictiva y marginal de la canción, aunque con más detalles y elementos. Estos chamacos conversan y dialogan de manera que forman una comunidad o fraternidad en la que comparten temas, ideas, sentimientos, temores y filosofía de vida. Su reflexión sobre su realidad en la vida urbana o suburbana llega a la conclusión que afirma lo que niegan y a la vez sugieren los lemas irónicos del Candide de Voltaire y de "La guaracha del Macho Camacho”, pues la conclusión ("Los chamacos hablan [...] de que esto es una encerrona disfrazá de felicidá”) refiere elusivamente el lema de Panglós: “este es el mejor de los mundos posibles”; y el de La guaracha: "La vida es una cosa fenomenal”. Estos muchachos habitan la esquina, hacen en ella su cultura y de ella su espacio vital, espacio que, como en "Las esquinas son”, convive al margen del orden y está caracterizado por aspectos no oficiales de la cultura en cuanto que refiere tanto a las drogas como a formas marginales del arte como el graffiti y los subterráneos, que alude tanto a recitales de poesía como a bailes y presentaciones de grupos de rock y de hip hop. 
Sin embargo, el poema termina negando la afirmación inicial pues "Los chamacos se fueron de la esquina a tiros. / Los chamacos calentaron el punto...”. Si la canción de Blades y Miranda terminaba con cierto dejo de nostalgia al presentar una distancia temporal entre la voz poética y las esquinas, el poema de Gallego sugiere la transformación del espacio de ocio en el centro de distribución de drogas que en la canción de Blades era presentado muy livianamente. En “Chamaco's corner” la esquina es transformada por la violencia y por la droga: la delimitación territorial inicial halló un macho más fuerte que desplazó a estos chamacos -con cuya conducta se asociaba y estimaba la voz poética-por otros. “Chamaco's corner”, entonces, coincide con una preferencia nostálgica por la esquina de esos chamacos marginales, pero de buen corazón y no asociados con la violencia del tráfico de drogas, sino con sus víctimas: los tecatos, los locos, y a los que mataron.

La esquina se recupera entonces mediante el poema: “Chamaco's corner” es el poema y la esquina en que Gallego mezcla y entrecruza sus viajes por la cultura callejera y la poesía, por la oralidad y la escritura, la música y la literatura. El lenguaje de Barrunto se distingue por su particular integración de la oralidad en la escritura, principalmente por construir hiatos de palabras separadas gramaticalmente, la incorporación del inglés y los apócopes, entre otros giros de la lengua oral puertorriqueña. Además, tanto el título del libro como el de varios poemas alude a canciones y grupos de música popular, en especial de la salsa. De esta forma, la poesía de Gallego se presenta como un cruce entre esos mundos que él mismo ve separados en la sociedad, pero que conforman las encrucijadas entre su origen, sus viajes, sus deseos y proyectos. Según los presenta en la "Introducción” de su disco compacto Teatro del barrio, donde contrasta su solicitud de entrada al Departamento de Drama de la Universidad de Puerto Rico con un "mundo real" al que paradójicamente llama "teatro del barrio", Gallego entonces intenta tanto en sus incursiones literarias como musicales situarse en esa esquina, doblando hacia la escritura y hacia la oralidad, hacia alusiones literarias y hacia referencias callejeras, a la música y a la poesía, como si decidir por una no significara renunciar a la otra. La esquina, como a los chamacos de su poema, le sirve para "estar para'o" y para doblar en cualquier dirección, no hacia un solo destino: a veces canta para complacer y a veces canta y escribe para provocar el pensamiento.

Las esquinas resultan espacios de moradas, encrucijadas y cruces. Se les estima como espacios en los cuales construir historias y delimitar territorios. Se les puede mirar románticamente como paisajes perdidos, socialmente como tránsitos y moradas o trascendentalmente como encrucijadas o conflictos culturales y sociales. Las letras de salsa y el poema de Gallego presentan a su vez una especie de lucha por "el espacio sonoro" como la que hubo en la década de 1960 entre la bachata y el merengue en República Dominicana, como recuerda Deborah Pacini Hernández. 
O se podría entender como encrucijada de la lucha por la esfera pública, según Melanie Pérez Ortiz observa de las interacciones entre literatura y cultura popular en Puerto Rico y México. Competencia en la que los músicos populares buscan legitimación entre la intelectualidad caribeña, así como estos intelectuales la han buscado entre la intelectualidad occidental y norteamericana, según la historia que traza Torres-Saillant. Para Pérez Ortiz, los escritores se apropian de la cultura popular intentando conquistar su público y su mercado, en una sociedad en la que la ciudad letrada pierde visibilidad y prominencia social frente a la cultura popular. Como en "Pedro Navaja”, estas culturas parecen encontrarse como en una esquina: a veces una mata o desplaza a la otra, como Blades mata a Pedro Navaja o como Gallego saca a los muchachos de la esquina; o ingenian un negocio que es simultáneamente explotación y protección de muchachas, como Isabel la Negra. A veces, simplemente, se encuentran, se miran, se saludan, se quedan parados y/o componen un son o escriben un verso; a veces una se queda y la otra pasa; se eluden, se juntan y/o siguen (¿su destino?) como en esta esquina.

\section{BiBLIOGRAFÍA}

Arce Bruguera, Arisel y Armando Ferrer Castro. El mundo de los orishas. La Habana: Unión, 1999.

Benítez Rojo, Antonio. La isla que se repite. Barcelona: Casiopea, 1998.

Certeau, Michel de. Practices of Everyday Life. Berkeley: U of California P, 1984.

Clifford, James. "Traveling Cultures”. Cultural Studies. Lawrence Grossberg et al. New York: Routledge, 1992. 96-116.

Deleuze, Gilles y Félix Guattari.A Thousand Plateaus. Capitalism and Schizophrenia. Minneapolis: U of Minnesota P, 1987.

Fiet, Lowell. "Walcott's Way: 'Do you know where you are’? 'At Crossroads in the Moonlight’”. Sargasso (1999): 103-13.

Fiske, John. Understanding Popular Culture. New York: Routledge, 1989.

Gates, Henry Louis. The Signifying Monkey. A Theory of African-American Literary Criticism. New York: Oxford UP, 1988.

Gelpí, Juan. Literatura y paternalismo en Puerto Rico. Río Piedras: Universidad de Puerto Rico, 1993.

Glissant, Édouard. Poetics of Relation. Ann Arbor: The U of Michigan P, 1997. González, José Raúl (Gallego). Barrunto. San Juan: Isla Negra, 2000.

Lamming, George. In the Castle of My Skin. Ann Arbor: The U of Michigan P, 1983. 
Martínez-San Miguel, Yolanda. Caribe Two Ways. Cultura de la migración en el Caribe insular hispánico. San Juan: Callejón, 2003.

Pacini Hernández, Deborah. "La lucha sonora: Dominican Popular Music in the post-Trujillo Era”. Latin American Music Review 12 (1991): 105-123.

Pedreira, Antonio S. Insularismo. San Juan: Plaza Mayor, 2001.

Pérez Ortiz, Melanie Ann. Between Lettered, Popular, and Mass Cultures: Intellectuals and the Public Sphere in Mexico and Puerto Rico: A Reading of the Works of Carlos Monsiváis, Cristina Pacheco, Edgardo Rodríguez Juliá and Ana Lydia Vega. Dissertation. Stanford University, 2000.

Quintero Herencia, Juan Carlos. La máquina de la salsa. Tránsitos del sabor. San Juan: Vértigo, 2005.

Quintero Rivera, Angel G. Salsa, sabor y control. Sociología de la música tropical. México: Siglo XXI, 1998.

Rondón, César Miguel. El libro de la salsa. Crónica de la música del Caribe urbano. Caracas: n.p., 1980.

Sánchez, Luis Rafael. La guagua aérea. Río Piedras: Cultural, 1994. “¡Jum!”. En cuerpo de camisa. Río Piedras: Antillana, 1975. 49-55.

Santos-Febres, Mayra. Nuestra Señora de la noche. Madrid: Espasa Calpe, 2006.

Torres-Saillant, Silvio. An Intellectual History of the Caribbean. New York: Palgrave, 2006.

Walcott, Derek. Dream on Monkey Mountain and Other Plays. New York: Noonday, 1970.

Wilson-Tagoe, Nana. "From Myth to Dialectic: History in Derek Walcott's Drama". Historical Thought and Literary Representation in West Indian Literature. Gainsville: U of Florida P, 1998. 169-81.

\section{DisCOGRAFÍA}

Blades, Rubén. Antecedente. Elektra/Asylum, 1988. AAD.

Colón, Willie \& Rubén Blades. Siembra. Fania Records, 1978. JMCD-537.

El Gran Combo de Puerto Rico. 30 Aniversario. Bailando con el mundo. Combo Records, 1992. Series 003498.

Gallego. Teatro del barrio. Machete Music, 2007.

Miranda, Ismael. En fa menor. Fania Records, 1974. SLP 00466.

Trío Matamoros. "El son de la loma”. 